\title{
Protective effects of curcumin against rat intestinal inflammation-related motility disorders
}

\author{
YANG YAO, RANYUAN LUO, SHU XIONG, CHANG ZHANG and YUKUN ZHANG \\ Department of Basic Medicine, Chongqing Three Gorges Medical College, Chongqing 404120, P.R. China
}

Received February 22, 2020; Accepted December 15, 2020

DOI: $10.3892 / \mathrm{mmr} .2021 .12030$

\begin{abstract}
Intestinal inflammation frequently occurs alongside dysmotility, which is characterized by altered myosin light chain phosphorylation levels. Curcumin, an active component from the ginger family, is reported to confer anti-inflammatory effects. However, the effects of curcumin on both diarrhea and constipation associated inflammation remains to be elucidated. The present study was designed to investigate the effects of curcumin on diarrhea and constipation and to determine the related mechanisms. Sprague-Dawley rats were used to establish diarrhea and constipation models via intracolonic acetic acid (4\%) instillation or cold water gavage for 2 weeks, respectively. Blood samples were collected to measure the serum levels of the cytokines TNF- $\alpha$ and IL-1 $\beta$ using ELISA kits. Western blotting was performed to measure NF- $\kappa \mathrm{B}$, RhoA, Rho-related kinase 2, phosphorylated $\mathrm{MLC}_{20}$, phosphorylated myosin phosphorylated target subunit 1, 130k Da-MLC kinase (MLCK), c-kit tyrosine kinase protein expression, and reverse transcription-quantitative PCR was conducted to measure MLCK expression levels. The results indicated that curcumin reversed the elevations in the pro-inflammatory cytokines IL- $1 \beta$ and TNF- $\alpha$ by inhibiting the NF- $\kappa$ B pathway in rats with diarrhea and constipation. The results also indicated that myosin light chain (MLC) phosphorylation in intestinal smooth muscle was positively and negatively associated with the motility of inflammation-related diarrhea and constipation in rats, respectively. Curcumin significantly reversed the increased MLC phosphorylation in the jejunum of the
\end{abstract}

Correspondence to: Professor Yukun Zhang, Department of Basic Medicine, Chongqing Three Gorges Medical College, 366 Tianxing Road, Bai'anba Street, Wanzhou, Chongqing 404120, P.R. China

E-mail: kunkun8580@qq.com

Abbreviations: DP, diarrhea-prominent; CP, constipationprominent; MLCK, myosin light chain kinase; MLC, myosin light chain; ROCK-II, Rho-associated kinase 2; MYPT1, myosin phosphates target subunit 1; IBD, inflammatory bowel disease; IBS, irritable bowel syndrome; MLCP, myosin light chain phosphatase; ICCs, interstitial cells of Cajal

Key words: curcumin, NF-кB, Rho kinase pathway, myosin light chain phosphorylation, inflammation rats with diarrhea, significantly enhanced the reductions in inflammatory mediators, including TNF- $\alpha$ and IL-1 $\beta$, of rats with constipation and significantly ameliorated the related hyper-motility and hypo-motility in rats with both diarrhea and constipation. In conclusion, the potential roles of the MLC kinase, c-kit tyrosine and Rho A/Rho-associated kinase 2 pathways, which are involved in curcumin-induced amelioration of inflammation-related diarrhea and constipation, were explored in the present study. Results from the present study suggested that curcumin has potential therapeutic value for treating intestinal inflammation and inflammation-related motility disorders.

\section{Introduction}

Curcumin (Fig. 1) is an active component found in turmeric, a rhizomatous herbaceous perennial plant of the ginger family (1). Curcumin is widely used as a culinary spice and food colorant (2) and is widely known to possess numerous beneficial health effects (3). Recent studies indicate that curcumin has a wide range of biological activities, including anti-inflammatory, antioxidant, anticancer and antimicrobial activities (4-6).

Intestinal hypermotility and hypomotility disorders are associated with inflammatory bowel disease (IBD) and other types of chronic gut inflammations, such as chronic enteritis and allergic colitis (7). For instance, infectious diarrhea and gastric stress ulcerations are characterized by hypermotility, whilst intestinal pseudo-obstruction, reflux esophagitis and ulcerative colitis are associated with hypomotility (8). Agents that possess the ability to ameliorate inflammation and inflammation-related hypomotility and hypermotility disorders are sorted after.

Irritable bowel syndrome (IBS) can be divided into diarrhea-predominant irritable bowel syndrome (IBS-D), constipation-predominant irritable bowel syndrome (IBS-C) and mixed irritable bowel syndrome (IBS-M) according to symptoms (9). Previous studies suggested that curcumin was beneficial for treating IBS-D, IBS-C and IBS-M $(10,11)$. Hyper- or hypomotility disorders have been observed in different types of IBS (12). However, the potential effects of curcumin on intestinal inflammation and inflammation-related motility disorders remain to be elucidated.

In the present study, it was hypothesized that curcumin ameliorates both intestinal inflammation and 
inflammation-related motility disorders by returning the levels of myosin light chain (MLC) phosphorylation in intestinal smooth muscles to normal levels. Both diarrhea-prominent (DP) hypermotility and constipation-prominent (CP) rat models were used to investigate this hypothesis. The successful establishment of DP and CP rat models was assessed not only by measuring the parameters of the incidence of diarrhea or constipation, but also by measuring the phosphorylation levels of MLC in the MLC kinase (MLCK) and Rho kinase signaling pathways in the rat jejunal smooth muscle. In addition, inflammatory markers, including serum TNF- $\alpha$ and IL-1 $\beta$ levels and $\mathrm{NF}-\kappa \mathrm{B}$ translocation to the nucleus, were measured to verify the extent of inflammation. The aforementioned parameters were also used to determine whether curcumin could ameliorate both intestinal inflammation and inflammation-related hypermotility in DP rats and hypomotility in CP rats, with aims of unraveling the mechanisms underlying the dual effects of curcumin.

\section{Materials and methods}

Animals. A total of 60 male Sprague-Dawley rats (age, 8-10 weeks; weight, 180-220 g) were obtained from the Experimental Animal Center, Dalian Medical University [Certificate of Conformity: no. SCXK (Liao) 2008-0002; Dalian, China]. The experimental protocol was approved by the Dalian Medical University Animal Care and Ethics Committee (Dalian, China) and the animal care and treatment were conducted in accordance with the National Institutes of Health Guide for Care and Use of Laboratory Animals (Publication no. 85-23, revised 1985) (13). The rats were housed one per cage, with all the animals housed in a room at $25^{\circ} \mathrm{C}$ with $60 \%$ humidity and 12 -h light/dark cycles. The rats were fed a pelleted diet ad libitum with free access to water and were fasted overnight before the experiment.

Drugs. Curcumin (Purify speciation, $\geq 99 \%$; cat. no. 458-37-7) was purchased from Shanghai Aladdin Biochemical Technology Co., Ltd. Unless otherwise indicated, all other chemicals were obtained from Sigma Aldrich, Merck KGaA.

Experimental models of diarrhea and constipation. Sprague-Dawley rats were randomly divided into two groups ( $\mathrm{n}=10$ per group): The DP and CP groups. The CP group included sham rats, $\mathrm{CP}$ rats and curcumin-treated $\mathrm{CP}$ rats. Similarly, the DP group included sham rats, DP rats and curcumin-treated DP rats. CP rats were established by daily gavage with cold water $\left(0-4^{\circ} \mathrm{C}\right)$ for 2 weeks, whilst the $\mathrm{CP}$ sham rats were treated by daily gavage with room-temperature water instead of cool water (13). DP rats were established by intracolonic acetic acid (4\%) instillation using an injector with hose for 2 weeks, whereas the DP sham rats received intracolonic saline instillation $(14,15)$. The body weights of rats in each group were recorded every 2 days. Fecal moisture content and granule number of each group were measured at day 2 and day 7 post-CP and -DP induction to determine whether the rat models were successfully established. After the successful establishment of the CP and DP rat models, the rats were treated with $200 \mathrm{mg} / \mathrm{kg}$ curcumin by gavage once daily. Following 7 days of consecutive administration, the rats were sacrificed.

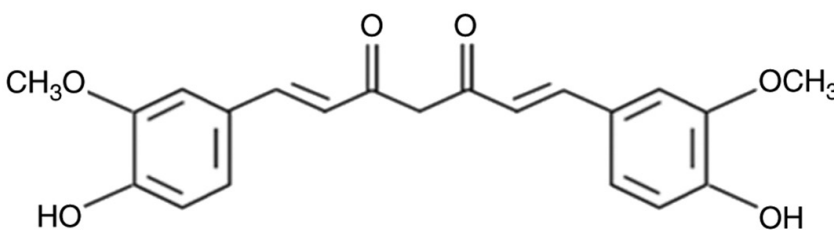

Figure 1. Chemical structure of curcumin.

Abdominal withdrawal reflex (AWR). The rats were first anesthetized with ether before a distension balloon catheter (6-Fr, $2 \mathrm{~mm}$ external diameter) was placed in the descending colon of mildly sedated rats. After waking up and adapting for $20 \mathrm{~min}$, a round of distention was applied for $10 \mathrm{sec}$, followed by a $20 \mathrm{sec}$ resting period. Distention at the various quantities $(0,0.25$ and $0.5 \mathrm{ml})$ were repeated twice, following which the balloon was deflated and withdrawn after measuring the AWR. Visceral sensory responses to rectal distension were quantified by scoring the AWR, as described previously (16). Briefly, 0 indicated no behavioral response during colorectal distention (CRD); 1 indicated an immobility response during $\mathrm{CRD}$ at the onset of the stimulus; 2 indicated mild contraction of the abdominal muscles without lifting the abdomen off the platform; 3 indicated strong contraction of the abdominal muscles without lifting the abdomen off the platform; and 4 indicated body arching, and lifting the pelvic structure and scrotum off the platform.

Hematoxylin and eosin $(H \& E)$ staining. The colon was fixed with $10 \%$ buffered formalin solution for $30 \mathrm{~min}$ at room temperature and then dehydrated in xylene followed by $75 \%$ ethanol overnight at room temperature, followed by paraffin embedding. Serial $4-\mu \mathrm{m}$ sections were cut. The sections were stained with hematoxylin and eosin for $1 \mathrm{~min}$ at room temperature for histologic analysis using a light microscope (magnification, x200).

Measurement of the serum TNF- $\alpha$ and IL-1 $\beta$ levels. Blood samples $(5 \mathrm{ml})$ were collected to measure the serum levels of the cytokines TNF- $\alpha$ and IL-1 $\beta$ using rat TNF- $\alpha$ Quantikine (cat. no. RTA00) and rat IL-1 $\beta$ Quantikine (cat. no. RLB00) ELISA kits (both R\&D Systems, Inc.).

Western blotting. The translocation and activity of NF- $\mathrm{KB}$, the content of Rho A, Rho-associated kinase 2 (ROCKII), phosphorylated (p)- $\mathrm{MLC}_{20}$, p-myosin phosphates target subunit 1 (MYPT1), $130 \mathrm{kDa}-\mathrm{MLCK}$ and the expression of c-kit tyrosine kinase in rat jejunal segments were determined using western blotting as previously described (16). Jejunal strips were teased along the natural line of cleavage from the longitudinal smooth muscle and the mucosal layer was removed. The samples were immediately weighed and minced on an ice-cold plate, following which the protein was extracted using a total protein extraction kit or a nuclear protein extraction kit (Nanjing KeyGen Biotech Co., Ltd.) and quantified by a bicinchoninic acid protein assay kit (Beyotime Institute of Biotechnology). Protein $(50 \mu \mathrm{g})$ from the tissue samples were separated by $7.5-20 \%$ SDS-PAGE and then transferred onto PVDF membranes. The PVDF membrane was probed 
Table I. Granules and moisture content of feces in rats.

\begin{tabular}{lcccc}
\hline & \multicolumn{2}{c}{ Day 2} & Day 7 \\
\cline { 2 - 5 } Groups & Granules, $\%$ & Moisture content, $\%$ & Granules, \% & Moisture content, \% \\
\hline DP sham & 100.0 & 100.0 & 100.0 & 100.0 \\
DP & $221.0 \pm 64.0^{\mathrm{a}}$ & $192.0 \pm 54.3^{\mathrm{a}}$ & $342.0 \pm 89.2^{\mathrm{a}}$ & $255.0 \pm 78.3^{\mathrm{a}}$ \\
DP + Cur & $120.0 \pm 32.2^{\mathrm{b}}$ & $175.0 \pm 45.5^{\mathrm{b}}$ & $102.8 \pm 61.2^{\mathrm{b}}$ & $154.0 \pm 74.9^{\mathrm{b}}$ \\
CP sham & 100.0 & 100.0 & 100.0 & 100.0 \\
CP & $31.0 \pm 20.2^{\mathrm{a}}$ & $45.0 \pm 14.4^{\mathrm{a}}$ & $25.0 \pm 12.3^{\mathrm{a}}$ & $33.0 \pm 21.7^{\mathrm{a}}$ \\
CP + Cur & $89.0 \pm 34.4^{\mathrm{b}}$ & $78.0 \pm 26.2^{\mathrm{b}}$ & $91.0 \pm 25.8^{\mathrm{b}}$ & $85.0 \pm 22.1^{\mathrm{b}}$ \\
\hline
\end{tabular}

The granules and moisture contents of feces from each sham control group are set to $100 \%$ (control). Data are expressed as the mean \pm SD (\% sham, $\mathrm{n}=6$ ). ${ }^{\mathrm{a}} \mathrm{P}<0.05$ vs. Sham and ${ }^{\mathrm{b}} \mathrm{P}<0.05$ vs. DP or CP. DP, diarrhea-prominent rats; $\mathrm{CP}$, constipation-prominent; Cur, curcumin.

Table II. Score of abdominal withdrawal reflex to colorectal distention.

\begin{tabular}{lccc}
\hline & & \multicolumn{2}{c}{ Volume of colon distention } \\
\cline { 3 - 4 } Groups & $\mathrm{N}$ & $0.25 \mathrm{ml}$ & $0.5 \mathrm{ml}$ \\
\hline CP related group & & & \\
Sham & 10 & $0.22 \pm 0.34$ & $2.70 \pm 0.33$ \\
Curcumin & 10 & $0.21 \pm 0.33$ & $2.40 \pm 0.34$ \\
CP & 10 & $0.30 \pm 0.42$ & $2.60 \pm 0.39$ \\
CP + Curcumin & 10 & $0.24 \pm 0.25$ & $2.50 \pm 0.28$ \\
DP related group & & & \\
Sham & 10 & $0.22 \pm 0.33$ & $2.50 \pm 0.29$ \\
Curcumin & 10 & $0.21 \pm 0.29$ & $2.60 \pm 0.26$ \\
DP & 10 & $0.23 \pm 0.25$ & $2.80 \pm 0.40$ \\
DP + Curcumin & 10 & $0.25 \pm 0.42$ & $2.48 \pm 0.59$ \\
\hline
\end{tabular}

$\mathrm{CP}$, constipation-prominent; DP, diarrhea-prominent.

with antibodies (all 1:1,000): IкB (cat. no. 40903; ProteinTech Group, Inc.), p65 (cat. no. 66535-1-Ig; ProteinTech Group, Inc.), p-p65 (cat. no. 3039s; Cell Signaling Technology, Inc.), Rho A (cat. no. 27213-1-AP; ProteinTech Group, Inc.), ROCKII (cat. no. 8236s; Cell Signaling Technology, Inc.), MLC20 (cat. no. 3672s; Cell Signaling Technology, Inc.), p-MLC20 (cat. no. 3675s; Cell Signaling Technology, Inc.), MYPT1 (cat. no. 2634; Cell Signaling Technology, Inc.), p-MYPT1 (cat. no. 3040T; Cell Signaling Technology, Inc.), MLCK (cat. no. 21642-1-AP; ProteinTech Group, Inc.) at $4^{\circ} \mathrm{C}$ overnight. A primary antibody against $\beta$-actin (cat. no. bs-0061R; BIOSS) or GAPDH (cat. no. 60004-1-Ig; ProteinTech Group, Inc.) for total protein and a primary antibody against histone-3 (cat. no. ab94817; Abcam) for nuclear protein was used as a loading control. Horseradish peroxidase-conjugated anti-rabbit/mouse IgG (cat. no. 33101ES60; Shanghai Yeasen Biotechnology Co., Ltd.) was used as secondary antibody at 1:1,000 for $60 \mathrm{~min}$ at room temperature. ECL (Beyotime Institute of Biotechnology) detection was performed for visualizing the protein bands, which were quantified by using the MultiSpectral imaging system (Analytik Jena AG).

Analysis of MLCK and gene expression by reverse transcription-quantitative $(R T-q) P C R$. Total RNA was extracted from the jejunal smooth muscle layer using TRIzol ${ }^{\circledR}$ reagent (cat. no. 15596026; Thermo Fisher Scientific, Inc.). cDNA was synthesized using the PrimeScript One Step RT-PCR kit (cat. no. RR055B; Takara Biotechnology Co.,Ltd.) at $45^{\circ} \mathrm{C}$ for $60 \mathrm{~min}$ and $72^{\circ} \mathrm{C}$ for $5 \mathrm{~min}$. Oligo (dT) primers (Promega Corporation) were used for RT-q PCR. The primers (Takara Biotechnology Co., Ltd.) were designed to detect the transcripts of MLCK according to the published sequences (2). The housekeeping gene $\beta$-actin served as a comparative reference. The primer sequences were as follows: MLCK forward, 5'-AATGGTGTT GCTGGAGATCGAGGT-3' and reverse, 5'-GCTGGATCA AATTGCGGTGGTTCA-3' and $\beta$-actin forward, 5'-GCA GGAGTACGATGAGTCCG-3' and reverse, 5'-ACGCAG CTCAGTAACAGTCC-3'. RT-qPCR was performed using SYBR Green-based fluorescence detection (cat. no. AQ142-21; TransStart Tip Green qPCR SuperMIX; TransGen Biotech Co., Ltd.). The PCR mixtures were denatured at $95^{\circ} \mathrm{C}$ for $30 \mathrm{sec}$, followed by 40 thermal cycles of $95^{\circ} \mathrm{C}$ for $5 \mathrm{sec}, 60^{\circ} \mathrm{C}$ for $35 \mathrm{sec}$ and $72^{\circ} \mathrm{C}$ for $30 \mathrm{sec}$. Relative gene expression was calculated using to the $2^{-\Delta \Delta \mathrm{Ct}}$ method (17).

Statistical analysis. Data are expressed as the mean \pm SD of $\geq \operatorname{six}$ independent repeats. Comparisons between two groups were performed using unpaired Student's t-test. To compare $\geq$ three groups, one-way ANOVA followed by Tukey's post hoc test was performed. The data were analyzed using SPSS (version 19.0; IBM Corp.). $\mathrm{P}<0.05$ was considered to indicate a statistically significant difference.

\section{Results}

Amelioration of diarrhea in DP rats and constipation in $C P$ rats. The DP rat model induced by acetic acid $(4 \% \mathrm{v} / \mathrm{v})$ instillation and the $\mathrm{CP}$ rat model induced by cool water $\left(4^{\circ} \mathrm{C}\right)$ gavage were used to model hyper- and hypomotility $(18,19)$. The successful establishment of inflammation-related diarrhea in the DP rats and inflammation-related constipation in 
A

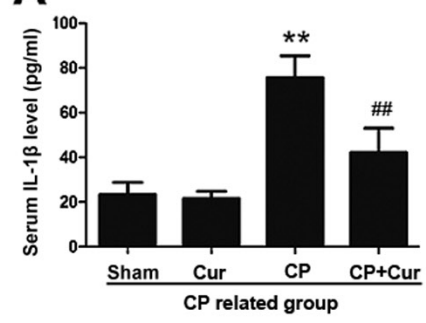

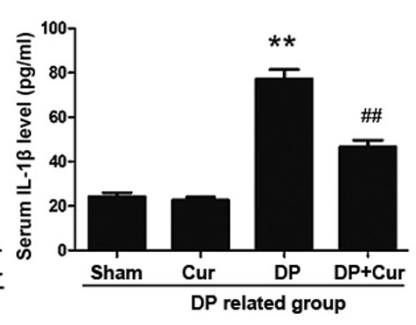

B

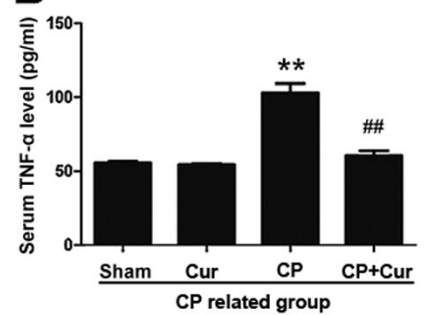

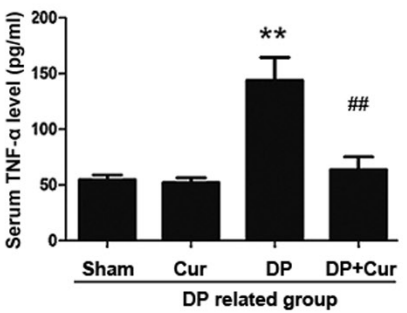

C
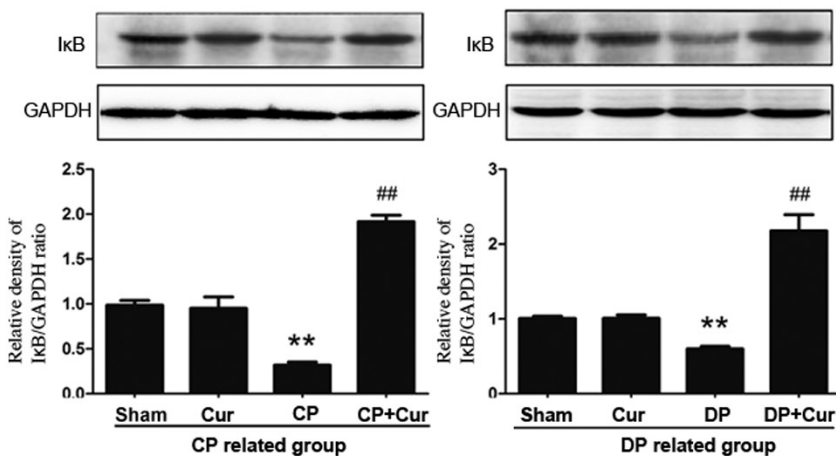

D
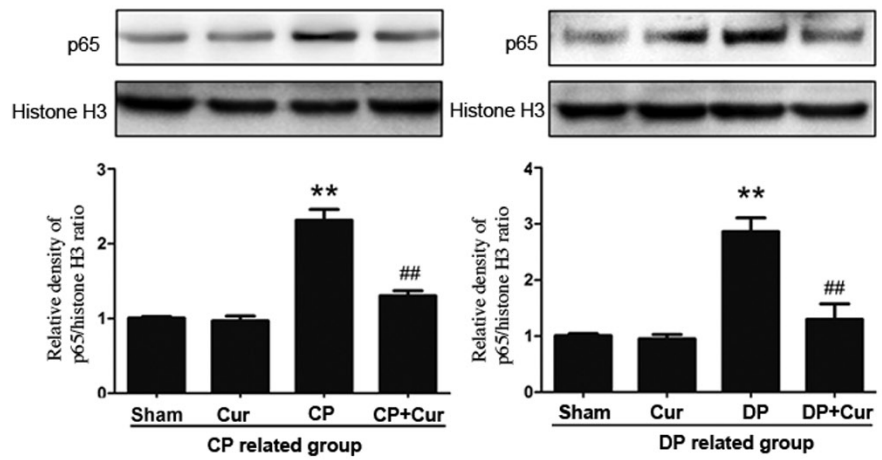
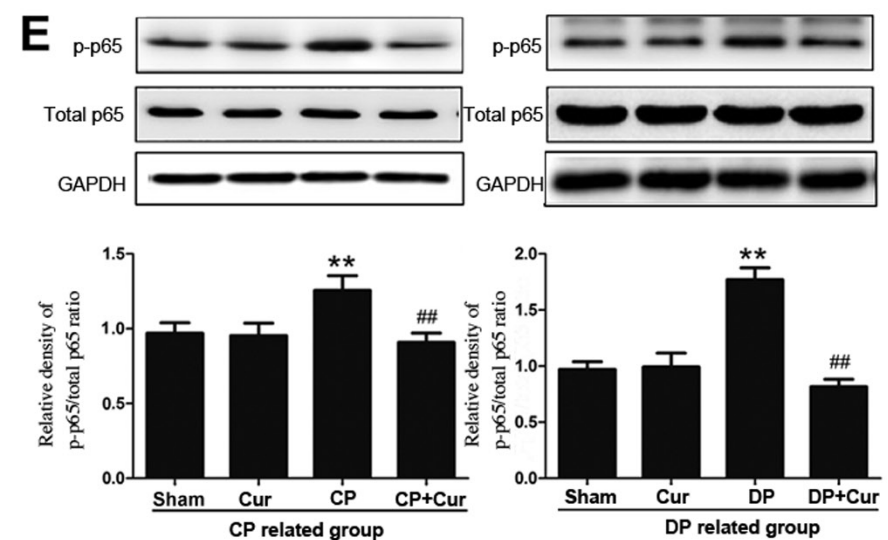

Figure 2. TNF- $\alpha$ and IL-1 $\beta$ release in DP and CP rat models and curcumin inhibits NF-kB p65 translocation in DP and CP models. Effects of curcumin on and (A) IL-1 $\beta$ and (B) TNF- $\alpha$ release in the serum of DP and CP rat models. ${ }^{* *} \mathrm{P}<0.05$ vs. Sham and ${ }^{\# \#} \mathrm{P}<0.05 \mathrm{CP}$ or DP. (C) Western blotting analysis for

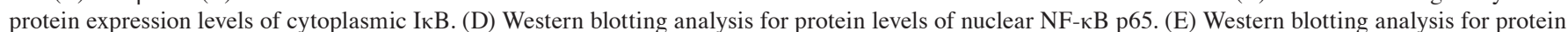
levels of phosphorylated $\mathrm{p} 65$. ${ }^{* *} \mathrm{P}<0.05$ vs. Sham and ${ }^{\# \#} \mathrm{P}<0.05$ vs. DP or CP. IL-1 $\beta$, interleukin-1 $\beta$; TNF- $\alpha$, tumor necrosis factor- $\alpha$; DP, diarrhea-prominent; $\mathrm{CP}$, constipation-prominent; Cur, curcumin; p, phosphorylated; IкB, inhibitor of NF-kB.

the $\mathrm{CP}$ rats was evaluated by determining the granules and moisture content of feces. On days 2 and 7, the granules and moisture contents in the feces from the $\mathrm{CP}$ and DP rats were significantly decreased and increased, respectively, compared with those in sham rats (Table I). Curcumin significantly reversed the reduction in granule and fecal moisture contents of the $\mathrm{CP}$ rats whilst also significantly reversing the increased granule and fecal moisture contents of the DP rats (Table I). This suggest that curcumin can ameliorate the symptoms of constipation and diarrhea in the $\mathrm{CP}$ and DP rats. Changes in AWR scores were next measured. The AWR score for all experimental groups during the recording period showed an increase in volume dependence by colorectal distention (colorectal distention, 0.25-0.5 ml water; Table II). However, no significant differences were observed among the groups. Additionally, no significant changes of colonic morphology were observed by H\&E staining (Fig. S1).

Amelioration of intestinal inflammation in both $C P$ and DP rats. The inflammatory cytokines TNF- $\alpha$ and IL-1 $\beta$, which serve important roles in the progression of both diarrhea and constipation, are associated with gut inflammation (20). In the present study, increased TNF- $\alpha$ and IL- $1 \beta$ levels were observed in the serum of the DP and CP rats compared with those in the Sham group (Fig. 2A and B), suggesting the presence of the inflammatory processes in both the DP and CP rats. Curcumin treatment significantly downregulated TNF- $\alpha$ and IL- $1 \beta$ release in both the DP and CP rats (Fig. 2A and B). 
A

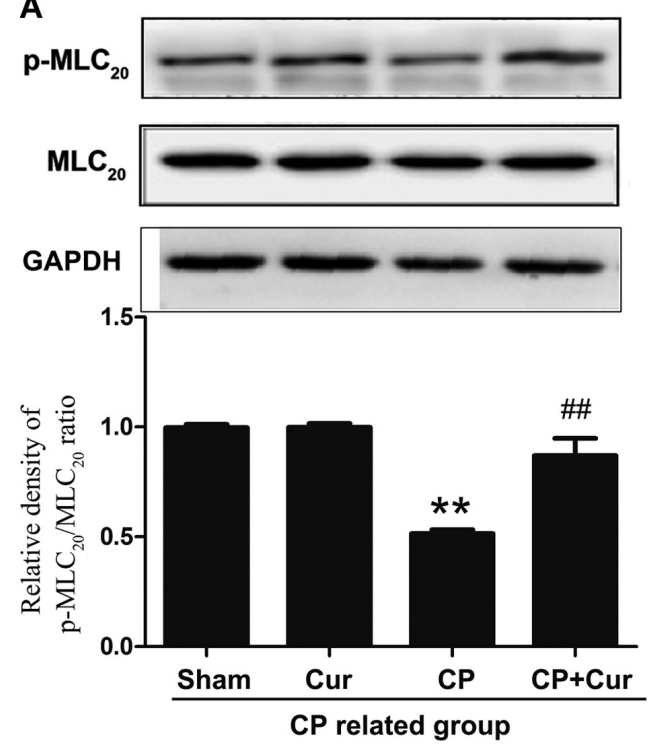

B

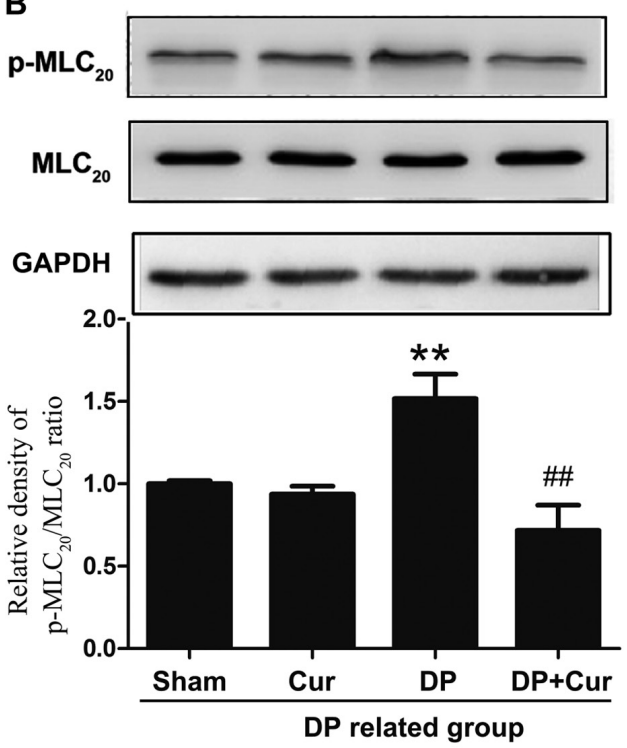

Figure 3. Effect of curcumin on MLC phosphorylation in curcumin treated diarrhea- DP and CP rat models. (A) Western blotting analysis of phosphorylation levels of $\mathrm{MLC}_{20}$ in CP rats. (B) Western blotting analysis of phosphorylation levels of $\mathrm{MLC}_{20}$ in DP rats. ${ }^{* *} \mathrm{P}<0.05$ vs. Sham and ${ }^{\# \#} \mathrm{P}<0.05$ vs. DP or CP. DP, diarrhea-prominent; CP, constipation-prominent; Cur, curcumin; MLC, myosin light chain; p-, phosphorylated.

A
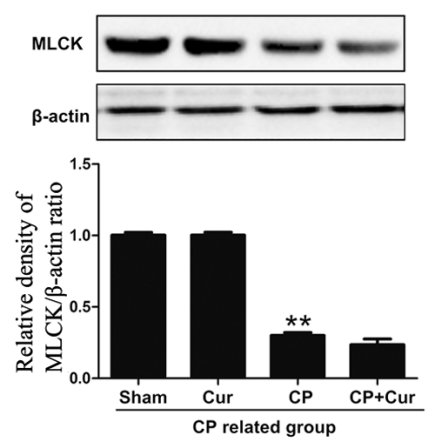

C
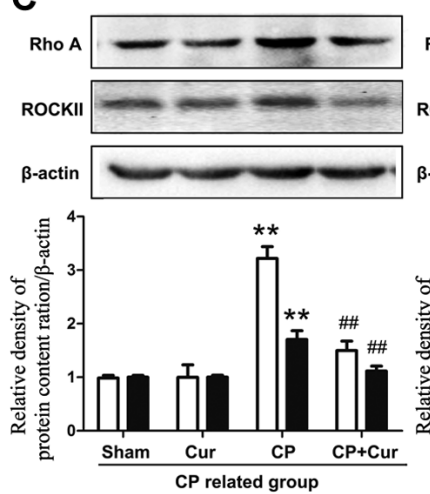
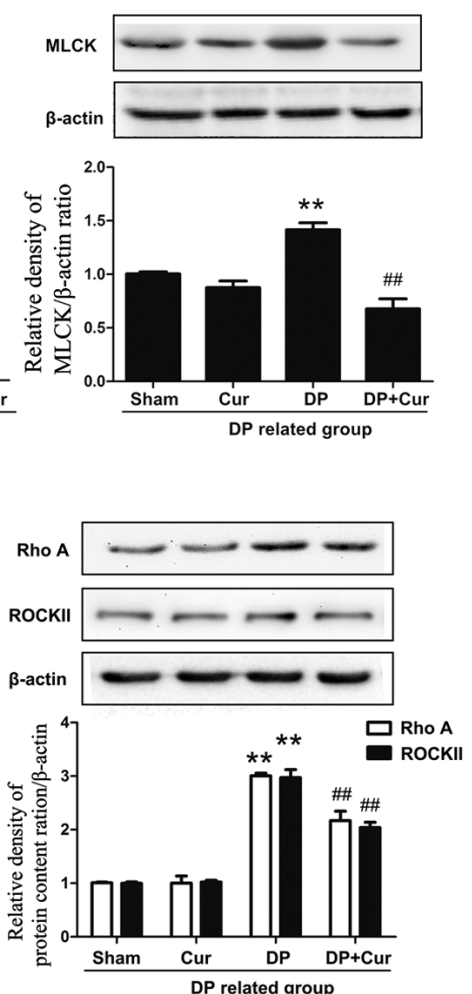

B

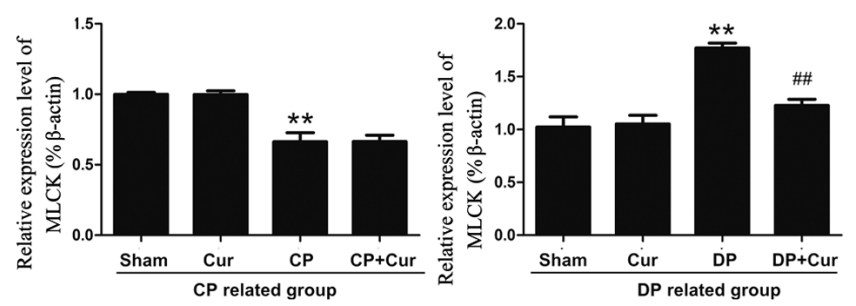

D
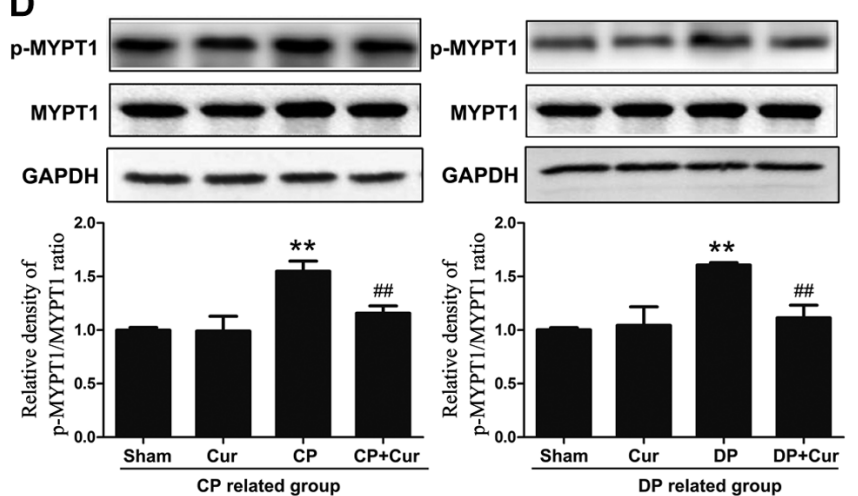

Figure 4. Effect of curcumin on long and short isoform MLCK, MLCK mRNA and MYPT1 expression. (A) Western blotting analysis of protein expression levels of MLCK. (B) MLCK mRNA expression levels by RT-qPCR analysis in CP and DP rats. (C) Western blot analysis of protein expression levels of RhoA and ROCKII in CP and DP rats. (D) Western blotting analysis for protein expression levels of phosphorylated MYPT1 and total MYPT1 in CP and DP rats. ${ }^{* *} \mathrm{P}<0.05$ vs. Sham and ${ }^{\# \#} \mathrm{P}<0.05$ vs. DP or CP. DP, diarrhea-prominent; $\mathrm{CP}$, constipation-prominent; Cur, curcumin; MLCK, myosin light chain kinase; ROCK II, Rho-associated kinase 2; MYPT1, myosin phosphate target subunit 1; RT-qPCR, reverse transcription-quantitative PCR; P-, phosphorylated.

The pro-inflammatory mediator NF- $\kappa \mathrm{B}$ is a nuclear transcription factor that serves a central role in inflammatory responses, which increases in TNF- $\alpha$ and IL-1 $\beta$ serum levels (1). NF- $\kappa B$ activation is characterized by I $\kappa B$ degradation, p65 nuclear translocation and increased p65 phosphorylation (20,21). Compared with the sham group, the NF- $\mathrm{kB}$ signaling pathway was activated in the CP and DP groups. The results of the present study indicated that curcumin inhibited the NF- $\mathrm{KB}$ 

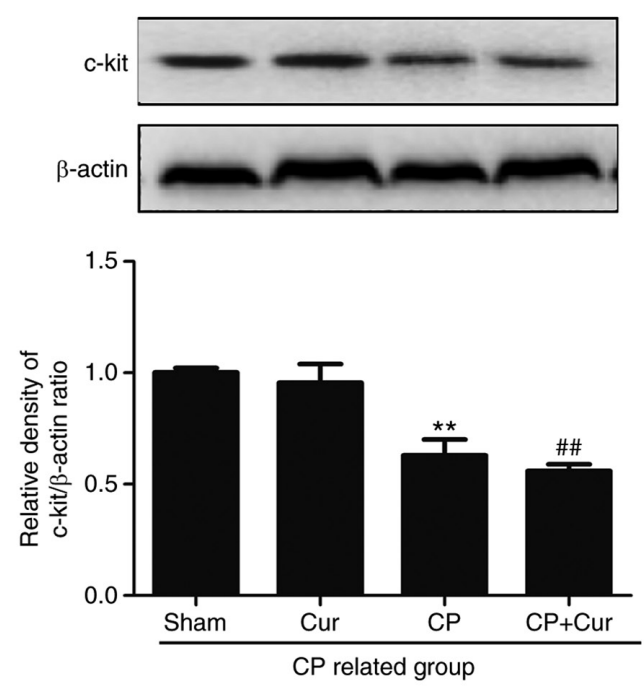
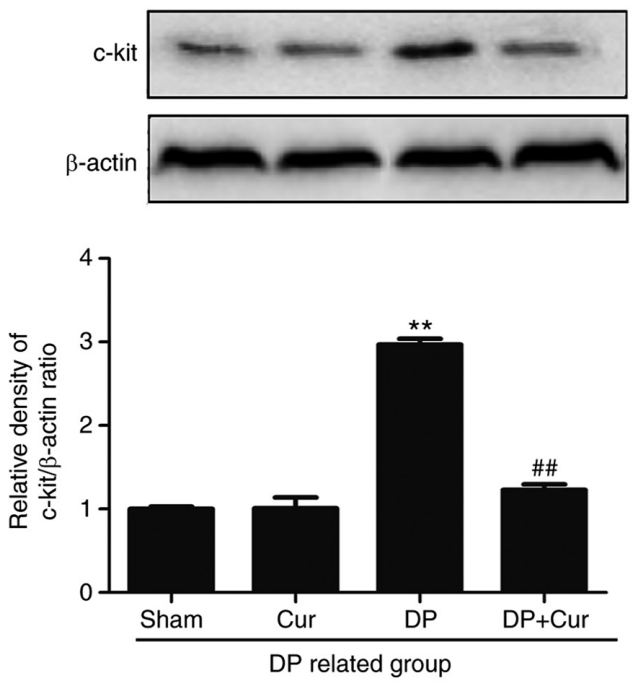

Figure 5. Effect of curcumin on protein expression levels of c-kit tyrosine kinase in the intestine of constipation-prominent and diarrhea-prominent related rat groups. ${ }^{* *} \mathrm{P}<0.05$ vs. Sham and ${ }^{\# \#} \mathrm{P}<0.05$ vs. DP or CP. DP, diarrhea-prominent; CP, constipation-prominent; Cur, curcumin.

pathway by significantly blocking IкB degradation (Fig. 2C), significantly inhibiting p65 nuclear translocation (Fig. 2D) and significantly reducing p65 phosphorylation (Fig. 2E) in the intestinal smooth muscle layer of the DP and CP rats. The aforementioned results indicated that curcumin reduced intestinal inflammation in DP and CP rats.

Curcumin induces MLC phosphorylation to normal levels through the Rho A/ROCK2/MYPT1 pathway. Intestinal motility is positively associated with MLC phosphorylation in the smooth muscle (22). Smooth muscle MLC phosphorylation is regulated by the relative activities of MLCK and myosin light chain phosphatase (MLCP) $(23,24)$. The results of the present study indicated significant reductions and increased $\mathrm{MLC}_{20}$ phosphorylation in the jejunal smooth muscle of the $\mathrm{CP}$ and DP rats, respectively (Fig. 3A and B). However, these were significantly reversed after 7 days of curcumin administration compared with those of the CP and DP rats (Fig. 3A and B).

To determine the associated mechanisms, the MLCK and MLCP activities were assessed. In the CP rats, reductions in $130 \mathrm{kDa}$ MLCK mRNA and protein expression levels were not significantly affected by the administration of curcumin (Fig. 4A and B). By contrast, in the DP rats, the increased $130 \mathrm{kDa}$ MLCK mRNA and expression levels were significantly reversed (Fig. 4A and B). Inhibition of MLCP activates the $\mathrm{Ca}^{2+}$ sensitivity pathway, leading to an increase in smooth muscle contraction (25). MYPT1 is the central subunit that regulates MLCP activity that can be activated by the Rho kinase and the NF- $\kappa$ B pathways upstream (26). Significantly increased levels of Rho A, ROCK II and phosphorylated MYPT1 expression were observed in the DP and CP rats compared with those in Sham rats (Fig. 4C and D). Curcumin treatment for 7 consecutive days significantly reversed the increased expression of Rho A, ROCK II and MYPT1 phosphorylation in both DP and CP rats (Fig. 4C and D).

Curcumin restores the normal function of interstitial cells of Cajal (ICCs). Damage to the ICCs may induce changes in the amplitude and duration of smooth muscle physical contractions (27). The activity of c-kit tyrosine kinase is associated with the function of ICCs in the gut (28). Significantly increased expression levels of c-kit tyrosine kinase in rat intestinal smooth muscle tissue in the DP rats and significantly decreased expression levels of c-kit tyrosine kinase in the $\mathrm{CP}$ rats were observed, compared with those in Sham rats (Fig. 5). Curcumin significantly reversed the increased expression levels of c-kit tyrosine kinase in the DP rats, but did not significantly upregulate the decreased expression levels of c-kit tyrosine kinase in the CP rats (Fig. 5), suggesting a potential role of curcumin in restoring the normal function of ICCs.

\section{Discussion}

Gut inflammatory disorders are associated with both diarrhea and constipation, with characteristics of intestinal dysmotility (29). For instance, diarrhea and constipation are associated with a phenotype or potentially inflammatory conditions such as IBS and IBD (30). Endogenous substances, including leptin (31) and oxytocin (32), have been reported to simultaneously participate in the regulation of gastrointestinal inflammation and motility. This suggests that ideal anti-inflammatory, naturally occurring and health-promoting compounds with low toxicity may exist that can simultaneously ameliorate both intestinal inflammation and inflammation-related diarrhea and constipation. Curcumin, which has been reported to be an anti-inflammatory and anticarcinogenic agent, is a potent inhibitor of $\mathrm{NF}-\kappa \mathrm{B}(33,34)$ that has been used to treat IBD (35). Curcumin has also been reported to interfere with colonic inflammation partly through the inhibition of chemokine expression and through the direct inhibition of neutrophil chemotaxis and chemokines (36). Based on the aforementioned information, it was hypothesized that curcumin could simultaneously modulate gut inflammation and motility disorders.

The major characteristics of the curcumin-induced bidirectional amelioration of diarrhea and constipation in DP and $\mathrm{CP}$ rats were as follows. Firstly, curcumin ameliorated the symptoms of diarrhea and constipation in the DP and CP rats. Secondly, curcumin significantly downregulated the expression 
of the inflammatory mediators TNF- $\alpha, \mathrm{IL}-1 \beta$ and $\mathrm{NF}-\kappa \mathrm{B}$ in the DP and CP rats. Thirdly, curcumin reversed the decreased $\mathrm{MLC}_{20}$ phosphorylation in the jejunal smooth muscle of the $\mathrm{CP}$ rats and reversed the increased $\mathrm{MLC}_{20}$ phosphorylation in the DP rats possibly through $\mathrm{Ca}^{2+}$-sensitive pathways.

The results of the present study demonstrated that curcumin ameliorated the symptoms and inflammation in the $\mathrm{CP}$ and DP rats, laying the foundation for further investigation on the effects of curcumin on smooth muscle motility-related phosphorylation. Smooth muscle contractility is regulated by the phosphorylation and dephosphorylation of $\mathrm{MLC}_{20}$, which is mediated by both the MLCK signaling and Rho kinase pathways. These pathways are known as $\mathrm{Ca}^{2+}$-dependent and $\mathrm{Ca}^{2+}$-sensitive pathways (37). Motility disorders, which are related to anomalous regulation of the $\mathrm{Ca}^{2+}$ and Rho A/ROCK II/MYPT1 pathways, are observed in gastrointestinal inflammatory disorders (38). The results of the present study indicated that increased IL- $1 \beta$, TNF- $\alpha$ and NF- $\kappa$ B are found during intestinal inflammation, which were reversed after curcumin administration in both $\mathrm{CP}$ and DP rats.

The decreased $\mathrm{MLC}_{20}$ phosphorylation in the $\mathrm{CP}$ rats and increased $\mathrm{MLC}_{20}$ phosphorylation in the DP rats were returned to normal levels by curcumin, indicating that curcumin benefits both inflammation-related hyper- and hypointestinal motility. The downregulation of the $130 \mathrm{kDa}$ MLCK mRNA and protein expression levels were observed only in the DP rats, unlike the upregulation of the $130 \mathrm{kDa}$ MLCK mRNA and protein expression levels in the CP rats. Instead, the increased expression levels of phosphorylated MYPT1 in the DP and CP rats were returned to normal levels after the administration of curcumin. In both CP and DP rats, curcumin inhibited the increased nuclear translocation of p65 and the $\mathrm{Ca}^{2+}$-sensitive pathway was activated as Rho A increased, followed by the upregulation of ROCK II and phosphorylated MYPT1. Phosphorylation of MYPT1 inhibits MLCP activity by directly interacting with protein phosphatase 1 catalytic subunit or by acting through a combination of molecular and biochemical mechanisms $(39,40)$. Changes in intracellular $\mathrm{Ca}^{2+}$ result in dysfunction of $\mathrm{Ca}^{2+}$-related proteins, such as c-kit tyrosine kinase, which is necessary for the function of Cajal cells; and MLCK, which promotes myosin phosphorylation (41). Results from the present study indicated that curcumin reversed the increased c-kit tyrosine kinase and MLCK expression levels, possibly through the inhibition of NF- $\kappa \mathrm{B}$ activity, in the DP rats. However, upregulation of MLCK in CP rats was not observed, suggesting that the curcumin-induced return of $\mathrm{MLC}_{20}$ phosphorylation to normal levels in the CP rats is not mediated by the MLCK signaling pathway, but more dependent on the $\mathrm{Ca}^{2+}$-sensitive pathway.

ICCs are pacemaker cells that generate electrical slow waves in the gastrointestinal tract and express c-kit tyrosine kinase, which is essential for ICC phenotype development and is required for spontaneous electrical and mechanical activities (42). Changes in the amplitude and duration of smooth muscle physical contractions are likely due to functional damage to ICCs (26). Phenotypic changes in ICCs were previously observed in a lipopolysaccharide-treated mouse model characterized by suppressed c-kit protein and mRNA expression (43), indicating that ICCs serve a vital role in motility changes during inflammation. The results of the present study indicated that the increased and decreased expression levels of c-kit tyrosine kinase in the $\mathrm{CP}$ and DP rats, respectively, were returned to normal by the administration of curcumin. This suggested that c-kit tyrosine kinase is involved in the curcumin-induced return of inflammation-related hyper- and hypojejunal contractility to normal.

Taken together, the present study demonstrated that curcumin returned the inflammation-related hyper-contractility in the DP rats and the hypo-contractility in the $\mathrm{CP}$ rats to normal through the NF- $\kappa \mathrm{B} / \mathrm{R}$ ho kinase pathway, in addition to c-kit and MLCK regulation. These results suggest that curcumin has potential value in relieving the inflammation-related alternating symptoms of gut dysmotility, where motility modulation during inflammatory conditions is based more on the $\mathrm{Ca}^{2+}$-sensitive pathway than the $\mathrm{Ca}^{2+}$-dependent pathway. However, although curcumin can inhibit gut inflammation, the role of curcumin on gut motility, the relationship between gut inflammation and motility disorders, as well as the detailed regulatory mechanism require further investigation. Since a number of studies have reported the anti-inflammatory effects of curcumin $(44,45)$, the present study investigated the role of curcumin in regulating gut motility based on the body status. Isolated smooth muscle segments and smooth muscle cells were used to investigate the mechanism of curcumin for intestinal inflammation and motility. The results demonstrated that curcumin inhibited smooth muscle contraction by blocking $\mathrm{Ca}^{2+}$ influx (46). Therefore, the effects of curcumin on intestinal motility is a highly complex process, including the regulation of the enteric nervous system and smooth muscle, but also the central nervous system.

\section{Acknowledgements}

Not applicable.

\section{Funding}

This work was supported by the Science and Technology Research Program of Chongqing Municipal Education Commission (grant nos. KJQN201902703 and KJZDK202002701), the Nursery Project of Chongqing Three Gorges Medical College (grant no. 2016mpxz21) and tbhe Natural Science Foundation of Chongqing (grant no. cstc2019jcyj-msxmX0299).

\section{Availability of data and materials}

The datasets used and/ or analyzed during the current study are available from the corresponding author on reasonable requests.

\section{Authors' contributions}

YZ conceptualized the study; YY and RL performed the experiments, analyzed the data and prepared the manuscript. SX and CZ conceived the study, provided the reagents and materials, and analyzed the data. All authors read and approved the final manuscript. 


\section{Ethics approval and consent to participate}

All animals received humane care in compliance with the Guide for the Care and Use of Laboratory Animals in China. The present study was approved by the Dalian Medical University Animal Care and Ethics Committee (approval no. 2019-4015).

\section{Patient consent for publication}

Not applicable.

\section{Competing interests}

The authors declare that they have no competing interests.

\section{References}

1. Lestari ML and Indrayanto G: Curcumin. Profiles Drug Subst Excip Relat Methodol 39: 113-204, 2014.

2. Prasad S, Gupta SC, Tyagi AK and Aggarwal BB: Curcumin, a component of golden spice: From bedside to bench and back. Biotechnol Adv 32: 1053-1064, 2014.

3. Aggarwal BB, Yuan W, Li S and Gupta SC: Curcumin-free turmeric exhibits anti-inflammatory and anticancer activities: Identification of novel components of turmeric. Mol Nutr Food Res 57: 1529-1542, 2013.

4. Homayouni A, Amini M, Sohrabi M, Varshosaz J and Nokhodchi A: Curcumin nanoparticles containing poloxamer or soluplus tailored by high pressure homogenization using antisolvent crystallization. Int J Pharm 562: 124-134, 2019.

5. Ridzuan NRA, Rashid NA, Othman F, Budin SB, Hussan F and Teoh SL: Protective role of natural products in cisplatin-induced nephrotoxicity. Mini Rev Med Chem 19: 1134-1143, 2019.

6. Wong KE, Ngai SC, Chan KG, Lee LH, Goh BH and Chuah LH: Curcumin Nanoformulations for Colorectal Cancer: A Review. Front Pharmacol 10: 152, 2019.

7. Ozaki H, Hori M, Kinoshita $\mathrm{K}$ and Ohama T: Intestinal dysmotility in inflammatory bowel disease: Mechanisms of the reduced activity of smooth muscle contraction. Inflammopharmacology 13: 103-111, 2005.

8. Du X, Allwood G, Webberley KM, Inderjeeth AJ, Osseiran A and Marshall BJ: Noninvasive diagnosis of irritable bowel syndrome via bowel sound features: Proof of concept. Clin Transl Gastroenterol 10: e00017, 2019.

9. Ng QX, Soh AYS, Loke W, Venkatanarayanan N, Lim DY and Yeo WS: A Meta-analysis of the clinical use of curcumin for irritable bowel syndrome (IBS). J Clin Med 7: 298, 2018.

10. Goulart RA, Barbalho SM, Lima VM, Souza GA, Matias JN, Araújo AC, Rubira CJ, Buchaim RL, Buchaim DV, Carvalho ACA and Guiguer EL: Effects of the use of curcumin on ulcerative colitis and Crohn's disease: A systematic review. J Med Food: Nov 5, 2020 (Epub of print).

11. Horii K, Ehara Y, Shiina T, Naitou K, Nakamori H, Horii Y, Shimaoka H, Saito S and Shimizu Y: Sexually dimorphic response of colorectal motility to noxious stimuli in the colorectum in rats. J Physiol, 2020 (Ahead of print).

12. Chen D, Xiong Y, Wang L, Lv B and Lin Y: Characteristics of emodin on modulating the contractility of jejunal smooth muscle. Can J Physiol Pharmacol 90: 455-462, 2012.

13. Zou BC, Dong L, Wang Y, Wang SH and Cao MB: Expression and role of 5-HT7 receptor in brain and intestine in rats with irritable bowel syndrome. Chin Med J (Engl) 120: 2069-2074, 2007.

14. La JH, Kim TW, Sung TS, Kang JW, Kim HJ and Yang IS: Visceral hypersensitivity and altered colonic motility after subsidence of inflammation in a rat model of colitis. World J Gastroenterol 9: 2791-2795, 2003.

15. Peng HY, Yeh CM, Cheng JK, Chau YP, Ruan T, Chen GD, Hsieh MC, Lai CY and Lin TB: Acute uterine irritation provokes colonic motility via transient receptor potential A(1)-dependent spinal NR2B phosphorylation in rats. Anesthesiology 120: 436-446, 2014.
16. Qi DB and Li WM: Effects of electroacupuncture on expression of c-fos protein in the spinal dorsal horn of rats with chronic visceral hyperalgesia. Zhong Xi Yi Jie He Xue Bao 10: 1490-1496, 2012.

17. Livak KJ and Schmittgen TD: Analysis of relative gene expression data using real-time quantitative PCR and the 2(-Delta Delta $\mathrm{C}(\mathrm{T}))$ method. Methods 25: 402-408, 2001.

18. Ohama T, Hori M, Momotani E, Iwakura Y, Guo F, Kishi H, Kobayashi S and Ozaki H: Intestinal inflammation downregulates smooth muscle CPI-17 through induction of TNF-alpha and causes motility disorders. Am J Physiol Gastrointest Liver Physiol 292: G1429-G1438, 2007.

19. Chen DP, Xiong YJ, Tang ZY, Yao QY, Ye DM, Liu SS and Lin Y: Characteristics of deslanoside-induced modulation on jejunal contractility. World J Gastroenterol 18: 5889-5896, 2012.

20. Kim HH, Kim DS, Kim SW, Lim SH, Kim DK, Shin TY and Kim SH: Inhibitory effects of Diospyros kaki in a model of allergic inflammation: Role of cAMP, calcium and nuclear factor- $\kappa$ B. Int J Mol Med 32: 945-951, 2013.

21. Zhang W, Sun J, Shen X, Xue Y, Yuan S and Wang X: Effect of PA-MSAH preprocessing on the expression of TLR-4-NF- $\kappa B$ pathway and inflammatory factors in the intestinal tract of rats with septic shock. Exp Ther Med 17: 2567-2574, 2019.

22. Sun G, Xing C, Zeng L, Huang Y, Sun X and Liu Y: Flemingia philippinensis Flavonoids Relieve Bone Erosion and Inflammatory Mediators in CIA Mice by Downregulating NF- $\mathrm{BB}$ and MAPK Pathways. Mediators Inflamm 2019: 5790291, 2019.

23. Xu Z, Liang H, Zhang M, Tao X, Dou D, Hu L and Kang T: Ardipusilloside-I stimulates gastrointestinal motility and phosphorylation of smooth muscle myosin by myosin light chain kinase. Korean J Physiol Pharmacol 21: 609-616, 2017.

24. Chang AN, Mahajan P, Knapp S, Barton H, Sweeney HL, Kamm KE and Stull JT: Cardiac myosin light chain is phosphor-

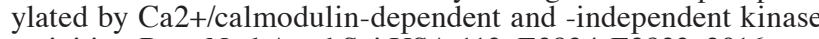
activities. Proc Natl Acad Sci USA 113: E3824-E3833, 2016.

25. Somlyo AP and Somlyo AV: Ca2+ sensitivity of smooth muscle and nonmuscle myosin II: Modulated by G proteins, kinases, and myosin phosphatase. Physiol Rev 83: 1325-1358, 2003.

26. Martelli L, Ragazzi E, di Mario F, Martelli M, Castagliuolo I, Dal Maschio M, Palu G, Maschietto M, Scorzeto M, Vassanelli S and Brun P: A potential role for the vanilloid receptor TRPV1 in the therapeutic effect of curcumin in dinitrobenzene sulphonic acid-induced colitis in mice. Neurogastroenterol Motil 19: 668-674, 2007.

27. Der T, Bercik P, Donnelly G, Jackson T, Berezin I, Collins SM and Huizinga JD: Interstitial cells of cajal and inflammation-induced motor dysfunction in the mouse small intestine. Gastroenterology 119: 1590-1599, 2000.

28. Jo HJ, Kim N, Nam RH, Kang JM, Kim JH, Choe G, Lee HS, Park JH, Chang H, Kim H, et al: Fat deposition in the tunica muscularis and decrease of interstitial cells of Cajal and nNOS-positive neuronal cells in the aged rat colon. Am J Physiol Gastrointest Liver Physiol 306: G659-G669, 2014.

29. Szałwińska P, Włodarczyk J, Spinelli A, Fichna J and Włodarczyk M: IBS-Symptoms in IBD patients-manifestation of concomitant or different entities. J Clin Med 10: 31, 2020.

30. Kamiya T, Shikano M, Tanaka M, Ozeki K, Ebi M, Katano T, Hamano S, Nishiwaki H, Tsukamoto H, Mizoshita T, et al: Therapeutic effects of biobran, modified arabinoxylan rice bran, in improving symptoms of diarrhea predominant or mixed type irritable bowel syndrome: A pilot, randomized controlled study. Evid Based Complement Alternat Med 2014: 828137, 2014.

31. Yarandi SS, Hebbar G, Sauer CG, Cole CR and Ziegler TR: Diverse roles of leptin in the gastrointestinal tract: Modulation of motility, absorption, growth, and inflammation. Nutrition 27: 269-275, 2011.

32. Welch MG, Margolis KG, Li Z and Gershon MD: Oxytocin regulates gastrointestinal motility, inflammation, macromolecular permeability, and mucosal maintenance in mice. Am J Physiol Gastrointest Liver Physiol 307: G848-G862, 2014.

33. Jobin C, Bradham CA, Russo MP, Juma B, Narula AS, Brenner DA and Sartor RB: Curcumin blocks cytokine-mediated NF-kappa $\mathrm{B}$ activation and proinflammatory gene expression by inhibiting inhibitory factor I-kappa B kinase activity. J Immunol 163: 3474-3483, 1999

34. Zhu T, Chen Z, Chen G, Wang D, Tang S, Deng H, Wang J, Li S, Lan J, Tong J, et al: Curcumin attenuates asthmatic airway inflammation and mucus hypersecretion involving a PPAR $\gamma$-Dependent $\mathrm{NF}-\kappa \mathrm{B}$ signaling pathway in vivo and in vitro. Mediators Inflamm 2019: 4927430, 2019. 
35. Torres J, Ellul P, Langhorst J, Mikocka-Walus A, Barreiro-de Acosta M, Basnayake C, Ding NJS, Gilardi D, Katsanos K, Moser G, et al: European crohn's and colitis organisation topical review on complementary medicine and psychotherapy in inflammatory bowel disease. J Crohns Colitis 13: 673-685e, 2019.

36. Larmonier CB, Midura-Kiela MT, Ramalingam R, Laubitz D, Janikashvili N, Larmonier N, Ghishan FK and Kiela PR: Modulation of neutrophil motility by curcumin: Implications for inflammatory bowel disease. Inflamm Bowel Dis 17: 503-515, 2011.

37. Álvarez-Santos MD, Álvarez-González M, Estrada-Soto S and Bazán-Perkins B: Regulation of myosin light-chain phosphatase activity to generate airway smooth muscle hypercontractility. Front Physiol 11: 701, 2020

38. Rajagopal S, Kumar DP, Mahavadi S, Bhattacharya S, Zhou R, Corvera CU, Bunnett NW, Grider JR and Murthy KS: Activation of $\mathrm{G}$ protein-coupled bile acid receptor, TGR5, induces smooth muscle relaxation via both Epac- and PKA-mediated inhibition of RhoA/Rho kinase pathway. Am J Physiol Gastrointest Liver Physiol 304: G527-G535, 2013.

39. Moreno-Dominguez A, Colinas O, El-Yazbi A, Walsh EJ, Hill MA, Walsh MP and Cole WC: $\mathrm{Ca}^{2+}$ sensitization due to myosin light chain phosphatase inhibition and cytoskeletal reorganization in the myogenic response of skeletal muscle resistance arteries. J Physiol 591: 1235-1250, 2013.

40. Grassie ME, Moffat LD, Walsh MP and MacDonald JA: The myosin phosphatase targeting protein (MYPT) family: A regulated mechanism for achieving substrate specificity of the catalytic subunit of protein phosphatase type $1 \delta$. Arch Biochem Biophys 510: 147-159, 2011.
41. Drumm BT, Rembetski BE, Messersmith K, Manierka MS, Baker SA and Sanders KM: Pacemaker function and neural responsiveness of subserosal interstitial cells of Cajal in the mouse colon. J Physiol 598: 651-681, 2020.

42. Somlyo AP and Somlyo AV: Smooth muscle: Excitation-contraction coupling, contractile regulation, and the cross-bridge cycle. Alcohol Clin Exp Res 18: 138-143, 1994.

43. Wei J, Li N, Xia X, Chen X, Peng F, Besner GE and Feng J: Effects of lipopolysaccharide-induced inflammation on the interstitial cells of Cajal. Cell Tissue Res 356: 29-37, 2014.

44. Fallahi F, Borran S, Ashrafizadeh M,Zarrabi A, Pourhanifeh MH, Khaksary Mahabady M, Sahebkar A and Mirzaei H: Curcumin and inflammatory bowel diseases: From in vitro studies to clinical trials. Mol Immunol 130: 20-30, 2020.

45. Qiao P, Ma J, Wang Y, Huang Z, Zou Q, Cai Z and Tang Y: Curcumin prevents neuroinflammation by inducing microglia to transform into the M2-phenotype via CaMKK $\beta$-dependent activation of the AMP-activated protein kinase signal pathway. Curr Alzheimer Res 17: 735-752, 2020.

46. Bardak H, Uğuz AC and Bardak Y: Curcumin regulates intracellular calcium release and inhibits oxidative stress parameters, VEGF, and caspase-3/-9 levels in human retinal pigment epithelium cells. Physiol Int 104: 301-315, 2017.

This work is licensed under a Creative Commons Attribution-NonCommercial-NoDerivatives 4.0 International (CC BY-NC-ND 4.0) License. 